[4] - "Radiation in a plasma: III, Metal boundaries," ibid., pp. 398-403.

[5] K. M. Chen, "Interaction of a radiating source with a plasmaeffect of an electroacoustic wave," Radiation Lab., University of Michigan, Ann Arbor, Tech. Rept. 4563-39-T, July 1963.

[6] J. R. Wait, "Radiation from sources immersed in compressible plasma media," Can. J. Phys., vol. 42, pp. 1760-1780, September 1964 .

[7] - "Theory of a slotted-sphere antenna immersed in a compressible plasma: I," Radio Science, J. Res. NBS/LSNC-URSI, vol. 68D, pp. 1127-1136, October 1964.

[8] "Theory of a slotted-sphere antenna immersed in a compressible plasma: II," ibid., pp. 1137-1143.

[9] J. A. Fejer, "Interaction of an antenna with a hot plasma and the theory of resonance probes," ibid., pp. 1171-1176, November 1964.

[10] J. R. Wait, "On radiation of electromagnetic and electroacoustic waves in plasma," Appl. Sci. Res., vol. 11, 1965.

[11] K. G. Balmain, "The impedance of a short dipole in a compressible plasma," Radio Science, $J$. Res. NBS/LSNC-LRSI, vol. 69D, pp. 559-566, April, 1965.
[12] L. Oster, "Linearized theory of plasma oscillations," Rev. Mod. Phys., vol. 32, pp. 141-168, January 1960.

[13] T. H. Stix, The Theory of Plasma Waves, New York, N. Y.: McGraw-Hill, 1962.

[14] W. P. Allis, S. J. Buchsbaum, and A. Bers, Waves in Anisotropic Plasmas. Cambridge, Mass.: MIT Press, 1962.

[15] J. R. Wait, "On radiation of electromagnetic and electroacoustic waves in plasma-part II," private communication.

[16] S. R. Seshadri, "Propagation constant for the current distribution along a cylindrical antenna immersed in a warm plasma," Applied Research Lab., Sylvania Electronic Systems, Waltham, Mass., Tech. Rept. 428, November 1964.

[17] R. W. P. King, Theory of Linear Antennas. Cambridge, Mass.: Harvard University Press, 1956.

[18] S. R. Seshadri, "Radiation in a warm plasma from an electric dipole with a cylindrical column of insulation," Applied Research Lab, Sylyania Electronic Systems, Waltham, Mass., Tech. Rept. 432, November 1964.

[19] H. A. Whale, "The excitation of electroacoustic waves by antennas in the ionosphere," J. Geophys. Res., vol. 68, pp. 415422, January 1963.

\title{
Antenna Radiation in a Moving Dispersive Medium
}

\author{
K. S. H. LEE AND C. H. PAPAS, MEMBER, IEEE
}

\begin{abstract}
The general problem of calculating the radiation field of an antenna immersed in a moving dispersive medium is formulated as an algebraic equation in wave-vector frequency space for the potential 4-vector in the rest frame of the antenna, and is solved in terms of a Green's function having the form of a one-dimensional integral. The special case where the moving medium is a homogeneous ionized gas (plasma) and the antenna is an oscillating dipole is studied in detail. It is found that the far-zone field is not transverse and the Poynting vector is not purely radial.
\end{abstract}

\section{INTRODUCTION}

(1) NE PRESSING problem of present-day antenna theory is the calculation of the radiation characteristics of an arbitrary antenna in a highvelocity wind of ionized gas. Theoretically, the problem can be thought of as having two interdependent parts, one being the hydrodynamic interaction of the antenna structure with the streaming fluid, and the other being the electromagnetic interaction of the emitted radiation with the moving medium. To solve this problem completely is a forbiddingly difficult task. However, when the antenna is so small that it does not markedly disturb the fluid, the problem becomes tractable. In this paper, we accordingly assume that the antenna is small enough

Manuscript received February 15, 1965. This research was supported by the U. S. Air Force, through the AF Office of Scientific Research, Office of Aerospace Research, under Contract AF49(638)1266.

The authors are with the Antenna Laboratory, California Institute of Technology, Pasadena, Calif. not to disturb the fluid, and therefore limit our discussion to the idealized problem of calculating the antenna radiation for the case where the ambient medium is a homogeneous, spatially unbounded, ionized gas moving at a uniform velocity with respect to the antenna. Clearly, this idealized problem is a generalization of a previously reported work [1] dealing with antenna radiation in the presence of moving simple media, for there the medium was restricted to be nondispersive whereas in the present problem the dispersive nature of the ionized gas cannot be ignored.

Although the present problem has not been treated in the literature, a simplified version of it, viz., the problem of Cerenkov radiation, has received considerable attention. As is well known, the Cerenkov radiation problem has been handled from two opposite points of view. In one the frame of reference is chosen to be at rest with respect to the medium, and in the other it is chosen to be at rest with respect to the charged particle. From the viewpoint of the rest frame of the medium, the problem amounts to one of solving the conventional inhomogeneous wave equation for the vector potential of the moving charge [2]. From the viewpoint of the rest frame of the charge, the medium appears to be moving past the charge, and to solve the problem Minkowski's electrodynamics of moving media must be invoked [3], [4]. Both points of view have proven equally fruitful. However, for the more general case where the source is 
an oscillating dipole in uniform motion, the problem has been analyzed in only the rest frame of the medium [5]. The problem we wish to solve in the present paper is essentially the complementary one of a stationary oscillating dipole immersed in a moving medium. Of course, the solutions of these two problems are related by Lorentz transformations, but it is more profitable to attack this complementary problem directly in the rest frame of the oscillating dipole. Accordingly, in the present paper we shall obtain the desired solution by considering the differential equation for the potential 4vector in the rest frame of the oscillating dipole and then solving this equation by Fourier transform methods.

There are two methods of deriving the required differential equation for the potential 4-vector [6]. Both stem from Minkowski's phenomenological electrodynamics of moving media which is based on the covariance of Maxwell's equations [7]. One method is to write Maxwell's equations and the constitutive relations in tensor form in wave-vector frequency space, and with the aid of the constitutive relations to eliminate one of the two field tensors in Maxwell's equations. Then, by expressing the remaining field tensor in terms of the potential 4 -vector and by invoking the generalized Lorentz condition, the required equation for the potential 4-vector can be obtained. The other method, which appears to be simpler, is to write in wave-vector frequency space the conventional inhomogeneous equations for the vector and scalar potentials in the rest frame of the medium and then to combine them to form a single equation for the potential 4-vector. The transformation of this equation from the rest frame of the medium to the rest frame of the source yields the required equation for the potential 4-vector. It is this simpler method that we shall use in the present paper.

In addition to the phenomenological approach already mentioned, there is the so-called atomistic approach that characterizes the medium by the polarization and magnetization vectors and describes the electromagnetic field by only two field vectors. The interrelations of these four vectors, as provided by Maxwell's equations, are free of constitutive parameters, but to solve them two extra equations that express the polarization and magnetization vectors in terms of the field vectors are needed. These extra equations are deduced by considering in the rest frame of the medium the interaction of the field vectors with the elementary constituents of the medium and then transforming the result to the rest frame of the source. Thus, in the rest frame of the source, Maxwell's equations involving only two field vectors can be obtained and solved directly. Although this atomistic approach is physically appealing, it is, by far, mathematically more complicated than the phenomenological approach, as is shown in the Appendix.

\section{INTEGRAL REPRESENTATION FOR THE POTENTIAL 4-Vector}

The problem we wish to solve is that of finding the radiation from a stationary monochromatic source completely immersed in a homogeneous isotropic dispersive medium that is moving past the source at uniform velocity $v$. We start by noting that in the rest frame $K^{\prime}$ of the medium the space-time Fourier transforms of the vector and scalar potentials satisfy the following algebraic equations:

$$
\begin{aligned}
& \left(-k^{\prime 2}+\frac{n^{\prime 2}}{c^{2}} \omega^{\prime 2}\right) A^{\prime}\left(\omega^{\prime}, k^{\prime}\right)=-\mu^{\prime} J^{\prime}\left(\omega^{\prime}, k^{\prime}\right) \\
& \left(-k^{\prime 2}+\frac{n^{\prime 2}}{c^{2}} \omega^{\prime 2}\right) \phi^{\prime}\left(\omega^{\prime}, k^{\prime}\right)=-\frac{1}{\epsilon^{\prime}} \rho^{\prime}\left(\omega^{\prime}, k^{\prime}\right)
\end{aligned}
$$

where $c$ is the vacuum velocity of light and the primes indicate that the quantities to which they are attached are measured in the $K^{\prime}$ frame. Clearly, $J^{\prime}\left(\omega^{\prime}, k^{\prime}\right)$ and $\rho^{\prime}\left(\omega^{\prime}, k^{\prime}\right)$ are the Fourier transforms of the current and charge densities of the source, and $A\left(\omega^{\prime}, k^{\prime}\right)$ and $\phi^{\prime}\left(\omega^{\prime}, k^{\prime}\right)$ are the Fourier transforms of the vector and scalar potentials. Since the medium is assumed to be only temporally dispersive in $K^{\prime}$, the permeability $\mu^{\prime}$, the dielectric constant $\epsilon^{\prime}$, and the index of refraction $n^{\prime}$ depend on the angular frequency $\omega^{\prime}$ of the radiation and not on the wave vector $k^{\prime}$.

As a consequence of the covariance of Maxwell's equations, $\left(k^{\prime}, i \omega^{\prime} / c\right),\left(A^{\prime}, i \phi^{\prime} / c\right)$, and $\left(J^{\prime}, i c \rho^{\prime}\right)$ are 4vectors. Using this fact we transform [1] (1) to the rest frame $K$ of the source and thus obtain the following algebraic equation for the potential 4 -vector $A_{\nu}(\omega, k)$ in terms of the source current density 4-vector $J_{\nu}(\omega, k)$ and the velocity 4-vector $U_{\nu}=(\gamma v, i \gamma c)$ where $\gamma$ $=\left(1-v^{2} / c^{2}\right)^{-1 / 2}$,

$$
\begin{aligned}
{\left[-k^{2}+\left(\omega^{2} / c^{2}\right)+\kappa^{\prime} \gamma^{2}(\omega-v \cdot k)^{2}\right] A_{\nu}(\omega, k) } \\
=-\mu^{\prime} J_{\nu}-\left(\mu^{\prime} \kappa^{\prime} / n^{\prime 2}\right) J_{\lambda} U_{\lambda} U_{\nu}
\end{aligned}
$$

The subscripts $\nu$ and $\lambda$ assume the values 1, 2, 3, 4, and the summation convention applies. This equation involves unprimed quantities, i.e., quantities measured in $K$, as well as the primed quantities $n^{\prime}\left(\omega^{\prime}\right), \epsilon^{\prime}\left(\omega^{\prime}\right)$, and $\kappa^{\prime}\left(\omega^{\prime}\right)$ which is a shorthand for $\left(n^{\prime 2}-1\right) / c^{2}$. Since $\omega^{\prime}=\gamma(\omega-v \cdot k)$, these primed quantities are actually functions of $\omega$ and $k$, and hence in the reference frame $K$ the medium appears to be not only temporally dispersive, but also spatially dispersive.

Solving (2) for $A_{p}(\omega, k)$ and taking the inverse spatial Fourier transform of the resulting equation, we obtain

$$
A_{s}(\omega, r)=\int \Gamma_{p \lambda}\left(r, r^{\prime}\right) J_{\lambda}\left(\omega, r^{\prime}\right) d V^{\prime} .
$$

Here $\boldsymbol{r}$ and $\boldsymbol{r}^{\prime}$ are position vectors in $K$ and the volume integration is with respect to the source position vector $\boldsymbol{r}^{\prime}$. The Green's function $\Gamma_{\nu \lambda}$ is given by 


$$
\begin{aligned}
\Gamma_{\nu \lambda}\left(r, \boldsymbol{r}^{\prime}\right)= & \frac{1}{8 \pi^{3}} \int \frac{\mu^{\prime} \delta_{p \lambda}+\left(\mu^{\prime} \kappa^{\prime} / n^{\prime 2}\right) U_{\nu} U_{\lambda}}{k^{2}-\left(\omega^{2} / c^{2}\right)-\kappa^{\prime} \gamma^{2}(\omega-v \cdot k)^{2}} \\
& \cdot \exp \left[i k \cdot\left(r-\boldsymbol{r}^{\prime}\right)\right] d^{3} k
\end{aligned}
$$

where $\delta_{v \lambda}$ is the Kronecker delta. Without loss of generality we choose $v$ to be directed along the $z$ axis of the Cartesian system $(x, y, z)$ that forms the spatial part of the reference frame $K$. This choice makes $\mu^{\prime}, n^{\prime}$, and $\kappa^{\prime}$ independent of the $x$ and $y$ components of $k$, viz., $k_{x}$ and $k_{y}$. Integrating with respect to $k_{x}$ and $k_{y}$, and recalling the well-known formulas

$$
\begin{aligned}
\frac{1}{2 \pi} \int_{0}^{2 \pi} e^{i u \rho \cos \left(x-\chi_{0}\right)} d \chi & =J_{0}(u \rho) \\
\int_{0}^{\infty} \frac{J_{0}(u \rho)}{u^{2}-u_{0}{ }^{2}} u d u & =\frac{i_{\pi}}{2} H_{0}{ }^{(1)}\left(u_{0} \rho\right)
\end{aligned}
$$

where $J_{0}$ is the zero-order Bessel function and $H_{0}^{(1)}$ is the zero-order Hankel function of the first kind, we obtain the following integral representation for the Green's function

$$
\begin{aligned}
\Gamma_{\nu \lambda}\left(r, r^{\prime}\right)= & \frac{i}{8 \pi} \int_{-\infty}^{\infty}\left[\mu^{\prime} \delta_{\nu \lambda}+\left(\mu^{\prime} \kappa^{\prime} / n^{\prime 2}\right) U_{\nu} U_{\lambda}\right] H_{0}^{(1)} \\
& \cdot[R \xi(s)] e^{i\left(z-z^{\prime}\right) s} d s
\end{aligned}
$$

where

$$
\begin{aligned}
R & =\sqrt{\left(x-x^{\prime}\right)^{2}+\left(y-y^{\prime}\right)^{2}}, \\
\xi(s) & =\sqrt{\omega^{2} / c^{2}+\kappa^{\prime} \gamma^{2}(\omega-v s)^{2}-s^{2}},
\end{aligned}
$$

and $k_{z}$ has been replaced by $s$.

Thus from a knowledge of the constitutive parameters of the medium one can find $\Gamma_{\nu \lambda}$ by evaluating the integral in (5), and knowing $\Gamma_{p \lambda}$ one can then with the aid of (3) obtain $A_{\nu}$ for any source distribution $J_{\lambda}$.

\section{Cerengov Radiation}

The foregoing result obtained can be readily applied to the problem of Cerenkov radiation where the source is a charged particle moving through a material medium. By setting $\omega=0, J=0, \rho\left(r^{\prime}\right)=q \delta\left(r^{\prime}\right)$ in (3) and (5) we obtain for the scalar and vector potentials in the rest frame of the charge the following expressions:

$$
\begin{aligned}
\phi & =\frac{q \gamma^{2}}{8 \pi i} \int_{-\infty}^{\infty} \frac{n^{\prime 2} \beta^{2}-1}{\epsilon^{\prime}} B_{0}^{(1)}\left(\rho s \gamma \sqrt{n^{2} \beta^{2}-1}\right) e^{i s z} d s \\
A_{z} & =\frac{q v \gamma^{2}}{8 \pi i c^{2}} \int_{-\infty}^{\infty} \frac{n^{\prime 2}-1}{\epsilon^{\prime}} H_{0}^{(1)}\left(\rho s \gamma \sqrt{n^{\prime 2} \beta^{2}-1}\right) e^{i s z} d s \\
A_{x} & =A_{y}=0
\end{aligned}
$$

where $\beta=v / c$ and $\rho^{2}=x^{2}+y^{2}$. These expressions agree with previous results [4] obtained in a less formal manner. Without further calculation one can see that there is radiation when $n^{\prime} \beta>1$ and no radiation when $n^{\prime} \beta<1$. This follows from the fact that for $n^{\prime} \beta<1$ the Hankel function behaves as

$$
\eta^{-1 / 2} e^{-\eta}\left(\eta=\rho s \gamma \sqrt{1-n^{\prime 2} \beta^{2}}\right)
$$

as $\rho \rightarrow \infty$.

\section{Dipole Radiation in Moving Ionized Gas}

Now we consider the case of an oscillating dipole immersed in an ionized gas moving past the dipole with uniform velocity $\mathbf{v}\left(=\boldsymbol{e}_{z} v\right)$. In the rest frame $K$ of the dipole, the dipole current is given by $J\left(\boldsymbol{r}^{\prime}\right)=-i \omega p \delta\left(\boldsymbol{r}^{\prime}\right)$ where $p$ is the dipole moment. In the rest frame $K^{\prime}$ of the medium we assume that $\mu^{\prime}=\mu_{0}$ and

$$
\epsilon^{\prime}=\epsilon_{0}\left(1-\omega_{p}^{\prime 2} / \omega^{\prime 2}\right)
$$

where

$$
\omega_{p}^{\prime 2}=N^{\prime} e^{2} /\left(n^{\prime} \epsilon_{0}\right),
$$

$N^{\prime}$ being the electron density, $m^{\prime}$ the electronic mass, $e$ the electronic charge, and $\mu_{0}, \epsilon_{0}$ the vacuum permeability and vacuum dielectric constant. Since $e, N^{\prime} / m^{\prime}$, $\epsilon_{0}$ are Lorentz invariants and since $\omega^{\prime}=\gamma(\omega-v \cdot k)$, we see that in $K$ we have

$$
\epsilon^{\prime}=\epsilon_{0}\left[1-\frac{\omega_{p}^{2}}{\gamma^{2}(\omega-v \cdot k)^{2}}\right] .
$$

Substituting $\mu_{0}$ for $\mu^{\prime}$ and expression (7) for $\epsilon^{\prime}$ in (5), we obtain

$$
\Gamma_{\nu \lambda}=\mu_{0} \delta_{\nu \lambda} G^{(0)}-\left(\mu_{0} / c^{2} \gamma^{2}\right) U_{\nu} U_{\lambda} G^{(1)} .
$$

Here

$$
\begin{aligned}
G^{(0)} & =\frac{e^{i k r}}{4 \pi r} \\
G^{(1)} & =\frac{i k_{p}^{2}}{8 \pi} \int_{-\infty}^{\infty} \frac{H_{0}^{(1)}\left(\rho \sqrt{k^{2}-s^{2}}\right) e^{i s z}}{\left(k_{0}-\beta s\right)^{2}-\left(1-\beta^{2}\right) k_{p}^{2}} d s
\end{aligned}
$$

where $k_{0}=\omega / c, k_{p}=\omega_{p} / c, k^{2}=k_{0}{ }^{2}-k_{p}{ }^{2}$, and $r$ is the the radial distance. Substituting expression (8) and $J\left(\boldsymbol{r}^{\prime}\right)=-i \omega p \delta\left(\boldsymbol{x}^{\prime}\right)$ into (3) we find that the potentials are given by

$$
\begin{aligned}
& A=-i \omega_{0} p G^{(0)}-\beta c \mu_{0} \mathbf{e}_{\varepsilon}\left(\boldsymbol{p} \cdot \nabla-i k_{0} \beta \boldsymbol{p} \cdot \mathbf{e}_{z}\right) G^{(1)} \\
& \phi=-\mu_{0} c^{2} \boldsymbol{p} \cdot \nabla G^{(0)}-\mu_{0} c^{2}\left(\boldsymbol{p} \cdot \nabla-i k_{0} \beta \boldsymbol{p} \cdot \mathbf{e}_{z}\right) G^{(1)} .
\end{aligned}
$$

We note that although these expressions for $A$ and $\phi$ do not satisfy the ordinary Lorentz conditions, they do satisfy the generalized Lorentz condition

$$
\nabla \cdot A-\left(i \omega / c^{2}\right) \phi-\kappa^{\prime} \gamma^{2}(\boldsymbol{v} \cdot \nabla-i \omega)(\boldsymbol{v} \cdot \boldsymbol{A}-\boldsymbol{\phi})=0
$$

as they must [6].

Having found $A$ and $\phi$, we get $E$ and $B$ from them through the use of $E=i \omega A-\nabla \phi$ and $B=\nabla \times A$. Thus from expressions (11) and (12) we obtain 


$$
\begin{aligned}
E= & \mu_{0} c^{2} p \cdot\left(u k_{0}^{2}+\nabla \nabla\right) G^{(0)}-i \beta \omega \mu_{0} c\left(e_{z} p \cdot \nabla+p \cdot e_{z} \nabla\right) G^{(1)} \\
& -\mu_{0} c^{2} \boldsymbol{p} \cdot\left(k_{0}^{2} \beta^{2} e_{z} e_{z}-\nabla \nabla\right) G^{(1)} \\
B= & i \omega \mu_{0} p \times \nabla G^{(0)}+\beta \mu_{0} c(\boldsymbol{p} \cdot \nabla) \mathrm{e}_{z} \times \nabla G^{(1)} \\
& -i \beta^{2} \omega \mu_{0}\left(p \cdot \mathrm{e}_{z}\right) e_{z} \times \nabla G^{(1)}
\end{aligned}
$$

where $\boldsymbol{u}=$ unit dyadic.

To find the Poynting vector $S$, which is a measure of the power flow, we need to calculate the magnetic intensity $\boldsymbol{H}$. We know that in wave-vector frequency space the constitutive relations are given by [7]

$$
\begin{aligned}
& D+\frac{1}{c^{2}} v \times H=\epsilon^{\prime}(E+v \times B) \\
& B-\frac{1}{c^{2}} v \times E=\mu_{0}(H-v \times D)
\end{aligned}
$$

and solving them for $H$ we get

$$
\begin{aligned}
H= & \frac{1}{\mu_{0}} B+\frac{\beta^{2}}{\mu_{0}}\left(u-e_{z} e_{z}\right) \cdot B \frac{k_{p}{ }^{2}}{\left(k_{0}-\beta s\right)^{2}} \\
& -\frac{\beta}{c \mu_{0}} \frac{k_{p}^{2}}{\left(k_{0}-\beta s\right)^{2}} \mathbf{e}_{z} \times E
\end{aligned}
$$

where expression (7) for $\epsilon^{\prime}$ has been used. Converting expressions (14) and (15) to wave-vector frequency space, we substitute the resulting expressions in to the right side of (17) and then take its inverse spatial Fourier transform to obtain $H$ in configuration space. Thus we have

$$
\begin{aligned}
H= & \frac{1}{\mu_{0}} B-\beta c e_{z} \times\left[p \cdot\left(k_{0}^{2} u+\nabla \nabla\right) G^{(2)}+p \cdot \nabla \nabla G^{(3)}\right] \\
& +i \beta^{2} \omega\left[\left(u-e_{z} e_{z}\right) \cdot p \times \nabla G^{(2)}+p \cdot e_{z} e_{z} \times \nabla G^{(3)}\right] \\
& +\beta^{3} c^{2}\left(u-e_{z} e_{z}\right) \cdot(p \cdot \nabla) e_{z} \times \nabla G^{(3)} \\
& -i \beta^{4} \omega\left(p \cdot e_{z}\right)\left(u-e_{z} e_{z}\right) \cdot e_{z} \times \nabla G^{(3)} .
\end{aligned}
$$

Here

$$
\begin{aligned}
G^{(2)}= & \frac{i k_{p}{ }^{2}}{8 \pi} \int_{-\infty}^{\infty} \frac{H_{0}{ }^{(1)}\left(\rho \sqrt{k^{2}}-s^{2}\right)}{\left(k_{0}-\beta s\right)^{2}} d s \\
G^{(3)}= & \frac{i k_{p}^{4}}{8 \pi} \\
& \cdot \int_{-\infty}^{\infty} \frac{H_{0}^{(1)}\left(\rho \sqrt{k^{2}-s^{2}}\right) e^{i s z}}{\left(k_{0}-\beta s\right)^{2}\left[\left(k_{0}-\beta s\right)^{2}-\left(1-\beta^{2}\right) k_{p}^{2}\right]} d s .
\end{aligned}
$$

Thus we see from expressions (14) and (18) that finding $E$ and $H$ is tantamount to evaluating the integrals $G^{(1)}, G^{(2)}$, and $G^{(3)}$ given, respectively, (10), (19), and (20). These integrals are difficult to evaluate in general. However, for the case of practical interest where $\beta \ll 1$ and $k_{0}>\gamma k_{p}$, we get the following approximate expressions

$$
\begin{aligned}
G^{(1)} & =\frac{k_{p}^{2}}{k^{2}}\left(1-2 i \beta \frac{k_{0}}{k^{2}} \frac{\partial}{\partial z}\right) G^{(0)}+0\left(\beta^{2}\right) \\
G^{(2)} & =\frac{k_{p}^{2}}{k_{0}^{2}}\left(1-2 i \beta \frac{1}{k_{0}} \frac{\partial}{\partial z}\right) G^{(0)}+0\left(\beta^{2}\right) \\
G^{(3)} & =\frac{k_{p}^{4}}{k_{0}^{2} k^{2}}\left(1-2 i \beta \frac{k_{0}^{2}+k^{2}}{k_{0} k^{2}} \frac{\partial}{\partial z}\right) G^{(0)}+0\left(\beta^{2}\right)
\end{aligned}
$$

where, as before, $G^{(0)}=\exp (i k r) / 4 \pi r$.

We now proceed to calculate the far-zone fields $E$, $H$, and the time-average far-zone Poynting vector $S$ of the oscillating dipole in the two independent cases where $p$ is 1) parallel, and 2) perpendicular to the motion of the ionized gas.

In case 1) where $p \| v$, i.e., $p=e_{z} p_{z}$, we obtain from (14), (18), and (21) the following expressions for the spherical components of the far-zone field:

$$
\begin{aligned}
& E_{r}=\beta \frac{k_{0} k_{p}^{2}}{\epsilon_{0} k} p_{z} \sin ^{2} \theta \frac{e^{i k r}}{4 \pi r} \\
& E_{\theta}=-\frac{k_{0}^{2}}{\epsilon_{0}} p_{z} \sin \theta \frac{e^{i k r}}{4 \pi r}-\beta \frac{k_{0} k_{p}^{2}}{\epsilon_{0} k} p_{z} \sin \theta \cos \theta \frac{e^{i k r}}{4 \pi r} \\
& E_{\phi}=0 \\
& H_{r}=H_{\theta}=0 \\
& H_{\phi}=-\omega k p_{z} \sin \theta \frac{e^{i k r}}{4 \pi r} .
\end{aligned}
$$

The spherical components of the time-average Poynting's vector of this field are given by

$$
\begin{aligned}
S_{r} & =\frac{\omega k_{0}{ }^{2} k}{32 \pi^{2} \epsilon_{0} r^{2}} p_{z}^{2} \sin ^{2} \theta+\beta \frac{\omega k_{0} k_{p}^{2}}{32 \pi^{2} \epsilon_{0} r^{2}} p_{z}^{2} \sin ^{2} \theta \cos \theta \\
S_{\theta} & =\beta \frac{\omega k_{0} k_{p}^{2}}{32 \pi^{2} \epsilon_{0} r^{2}} p_{z}^{2} \sin ^{3} \theta \\
S_{\phi} & =0\left(\beta^{2}\right) .
\end{aligned}
$$

In case 2) where $p \perp_{v}$, i.e., $p=e_{x} p_{x}$, we similarly obtain the following expressions for the spherical components of the far-zone field:

$$
\begin{aligned}
& E_{r}=-\beta \frac{k_{0} k_{p}^{2}}{\epsilon_{0} k} p_{x} \sin \theta \cos \theta \cos \phi \frac{e^{i k r}}{4 \pi r} \\
& E_{\theta}=\frac{k_{0}^{2}}{\epsilon_{0}} p_{x} \cos \theta \cos \phi \frac{e^{i k r}}{4 \pi r}-\beta \frac{k_{0} k_{p}^{2}}{\epsilon_{0} k} p_{x} \sin ^{2} \theta \cos \phi \frac{e^{i k r}}{4 \pi r} \\
& E_{\phi}=-\frac{k_{0}^{2}}{\epsilon_{0}} p_{x} \sin \phi \frac{e^{i k r}}{4 \pi r}
\end{aligned}
$$

$H_{r}=-\beta c k_{p}^{2} p_{x} \sin \theta \sin \phi \frac{e^{i k r}}{4 \pi r}$

$H_{\theta}=\omega k p_{x} \sin \phi \frac{e^{i k r}}{4 \pi r}-\beta c k_{p}{ }^{2} p_{x} \cos \theta \sin \phi \frac{e^{i k r}}{4 \pi r}$

$H_{\dot{\phi}}=\omega k p_{x} \cos \theta \cos \phi \frac{e^{i k r}}{4 \pi r}-\beta c k_{p}^{2} p_{x} \cos \phi \frac{e^{i k r}}{4 \pi r}$. 
The spherical components of the Poynting's vector of this field are

$$
\begin{aligned}
S_{r} & =\frac{\omega k_{0}^{2} k}{32 \pi^{2} \epsilon_{0} r^{2}} p_{x}^{2}\left(\cos ^{2} \theta \cos ^{2} \phi+\sin ^{2} \phi\right) \\
& -\beta \frac{\omega k_{0} k_{p}^{2}}{32 \pi^{2} \epsilon_{0} r^{2}} p_{x}^{2}\left(1+\sin ^{2} \theta \cos ^{2} \phi\right) \cos \theta \\
S_{\theta} & =\beta \frac{\omega k_{0} k_{p}^{2}}{32 \pi^{2} \epsilon_{0} r^{2}} p_{x}^{2}\left(\cos ^{2} \theta \cos ^{2} \phi+\sin ^{2} \phi\right) \sin \theta \\
S_{\phi} & =0\left(\beta^{2}\right) .
\end{aligned}
$$

Examination of the expressions (22) and (24) shows that in the rest frame of the source the far-zone fields are not TEM, in contradistinction to the case where the medium is stationary with respect to the source. Moreover, from expressions (23) and (25) we see that the Poynting vector has a nonzero peripheral component in addition to a radial one, and is tilted against the direction of flow of the gas, as shown schematically in Figs. 1 and 2.

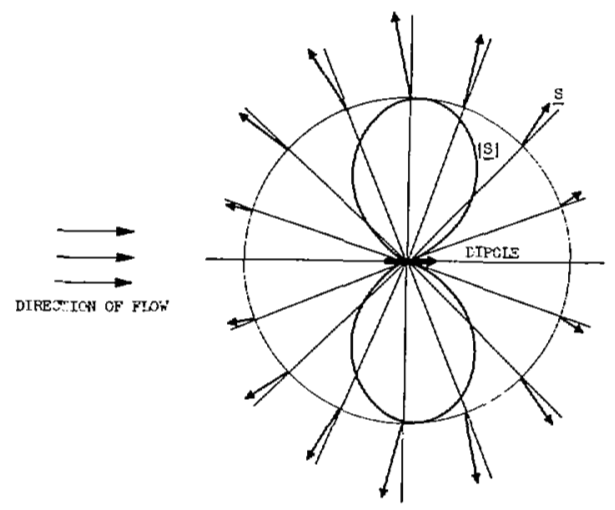

Fig. 1. Schematic diagram of power radiation pattern $|S|$ and far-zone Poynting's vector $S$ for case 1) $\boldsymbol{p}_{l}^{\|} \boldsymbol{v}$.

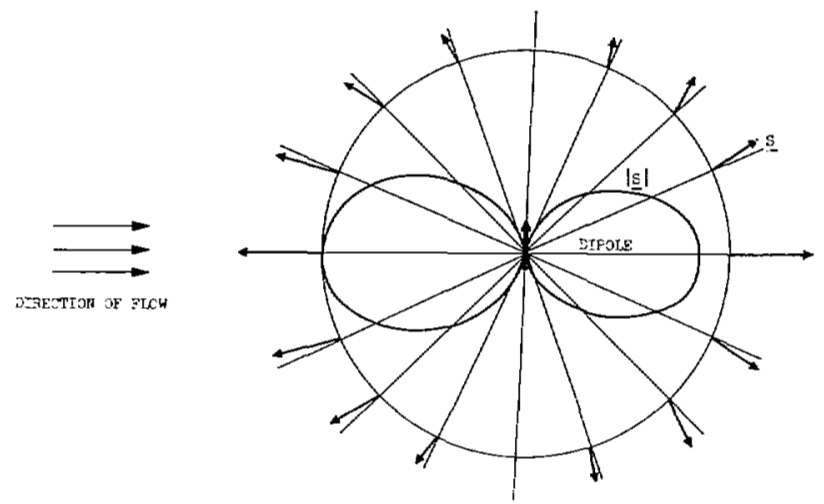

Fig. 2. Schematic diagram of power radiation pattern $|S|$ and far-zone Poynting's vector $S$ for case 2) $p \perp v$.

\section{Appendix}

The purpose of this Appendix is to show that the results obtained above using the phenomenological approach can also be derived from atomistic considerations. In the atomistic method Maxwell's equations in the rest frame $K$ of the source take the form

$$
\begin{aligned}
& \nabla \times E=-\frac{\partial}{\partial t} B \\
& \nabla \times B=\mu_{0} \epsilon_{0} \frac{\partial}{\partial t} E+\mu_{0} \frac{\partial}{\partial t} P+\mu_{0} \nabla \times M+\mu_{0} J
\end{aligned}
$$

where $P$ and $M$ are, respectively, the polarization and magnetization vectors of the medium. To find $P$ and $M$ in terms of $E$ and $B$ we first calculate $P^{\prime}$ and $M^{\prime}$ in the rest frame $K^{\prime}$ of the medium. Assuming the field to be so weak that the velocity acquired by an electron is small compared to the vacuum speed of light, we have the equation of motion

$$
m^{\prime} \frac{d}{d t^{\prime}} v^{\prime}=-e E^{\prime}
$$

where all the primed quantities are referred to the rest frame $K^{\prime}$ of the medium. Since $P^{\prime}$ is defined by the relation $\left(d / d t^{\prime}\right) P^{\prime}=-N^{\prime} e v^{\prime}$, where $N^{\prime}$ is the number of electrons per unit volume, we see from (27) that $P^{\prime}$ is related to $E^{\prime}$ as follows:

$$
\frac{d^{2}}{d t^{\prime 2}} P^{\prime}=\frac{N^{\prime} e^{2}}{m^{\prime}} E^{\prime} .
$$

Also we note that

$$
M^{\prime}=0 .
$$

With the aid of the relations [7]

$$
\begin{aligned}
\frac{d}{d l^{\prime}} & =\gamma\left(\frac{\partial}{\partial t}+v \cdot \nabla\right) \\
\frac{N^{\prime} e^{2}}{m^{\prime}} & =\frac{N e^{2}}{m}=\epsilon_{0} \omega_{p}^{2} \\
P & =\left[\gamma u+(1-\gamma) \frac{v v}{v^{2}}\right] \cdot P^{\prime} \\
M & =\gamma P^{\prime} \times v \\
E^{\prime} & =\gamma(E+v \times B)+(1-\gamma) \frac{v v}{v^{2}} \cdot E
\end{aligned}
$$

we obtain from (28) and (29) the following representation for $P$ and $M$ in wave-vector frequency space:

$$
\begin{aligned}
& P=-\frac{\epsilon_{0} \omega_{p}{ }^{2}}{(\omega-k \cdot v)^{2}}\left(E+v \times B-\frac{v V}{c^{2}} \cdot E\right) \\
& M=\frac{\epsilon_{0} \omega_{p}{ }^{2}}{(\omega-k \cdot v)^{2}} v \times(E+v \times B) .
\end{aligned}
$$

In wave-vector frequency space (26) becomes

$$
\begin{aligned}
& k \times E=\omega B \\
& k \times B=-\mu_{0} \epsilon_{0} \omega E-\mu_{0} \omega P+\mu_{0} k \times M-i \mu_{0} J
\end{aligned}
$$

so, by eliminating $P$ and $M$ from them by use of (31) we obtain two equations involving only two field vectors $E$ and $B$. At this stage one may introduce the potentials $A$ and $\phi$ through the relations $E=i \omega A-i k \phi, B=i k \times A$ 
with the generalized Lorentz condition (13), and thus obtain (2). Here, however, we shall work directly with the field vectors and, of course, arrive at the same results.

To illustrate the procedure let us work out the case where $\beta \ll 1$. Taking $v$ to be directed along the $z$ axis and neglecting terms higher than the first power of $\beta$, we obtain from (32) and (31) the following:

$$
\Gamma \cdot E=-i \omega \mu_{0} J
$$

where

$$
\boldsymbol{\Gamma}=\left(\begin{array}{c}
k_{x}^{2}-k^{2}+k_{0}^{2}-k_{p}^{2} \\
k_{x} k_{y} \\
k_{x} k_{z}-v \omega^{-1} k_{x} k_{p}^{2}
\end{array}\right.
$$

$$
\begin{gathered}
k_{x} k_{y} \\
k_{y}{ }^{2}-k^{2}+k_{0}{ }^{2}-k_{p}{ }^{2} \\
k_{y} k_{z}-v \omega^{-1} k_{y} k_{p}{ }^{2}
\end{gathered}
$$
leads to (22) for $E$.

The inverse spatial Fourier transform of this equation

Let us consider just the case where $p \| v$, i.e.,

$$
J=-i \omega p_{z} \delta(t) e_{2}
$$

Then (33) yields

$$
E=-\omega^{2} \mu_{0} p_{z} \Gamma^{-1} \cdot e_{z}
$$

Here $\Gamma^{-1}$ is the inverse of $\boldsymbol{\Gamma}$. Finding $\Gamma^{-1}$ from (34) and substituting in to (35) we get

\section{REFERENCES}

[1] K. S. H. Lee and C. H. Papas, J. Math. Phys., vol 5, p 1668, 1964.

[2] I. Frank and Ig. Tamm, C. R. Acad.Sci. (USSR), vol 14, p 109, 1937.

[3] K. M. Watson and J. M. Jauch, Phys. Rev, vol 75, p 1249, 1949.

[4] B. D. Nag, and A. M. Sayied, Proc. Roy. Soc. (London), vol A235, p 544, 1956.

[5] I. Frank, J. Phys. (USSR), vol 2, p 49, 1943.

[6] C. H. Papas, Theory of Electromagnetic Wave Propagation. New York: McGraw-Hill, ch 7, 1965.

[7] W. Pauli, Theory of Relativity. New York: Pergamon Press, 1958. 